\title{
ANGULAR DISTRIBUTION OF SOLUTIONS OF HIGHER ORDER LINEAR DIFFERENTIAL EQUATIONS
}

\author{
Zhaojun Wu and Daochun Sun
}

\begin{abstract}
In this paper, we study the location of zeros and Borel direction for the solutions of linear homogeneous differential equations$$
f^{(n)}+A_{n-1}(z) f^{(n-1)}+\cdots+A_{1}(z) f^{\prime}+A_{0}(z) f=0
$$

with entire coefficients. Results are obtained concerning the rays near which the exponent of convergence of zeros of the solutions attains its Borel direction. This paper extends previous results due to S. J. Wu and other authors.
\end{abstract}

\section{Introduction and statement of results}

In this paper, the term meromorphic function will mean meromorphic in the whole complex plane $\mathbb{C}$. We shall assume that the reader is familiar with the standard notation of Nevanlinna theory and complex differential equation (see [4] or [6]). The study of the angular distribution for meromorphic function was started by Julia. In 1919, Julia introduced the concept of Julia direction and showed that every transcendental entire function has at least one Julia direction. This result is a refinement of Picard's theorem. In order to have a similar refinement for Borel's theorem, a more refined notion of Borel direction was introduced by Valiron in 1928. Recently, J. H. Zheng [14] introduced a new direction for meromorphic function namely $T$ direction, that attains the Nevanlinna second fundamental theorem (see [3] or [14]). Now, we recall the definition of Borel direction as following.

Suppose that $g(z)$ is a meromorphic function of order $\rho(0<\rho \leq \infty)$. A ray $\arg z=\theta$ is called a Borel direction of order $\rho$ for $f$ if for every $0<\varepsilon<\frac{\pi}{2}$,

$$
\limsup _{r \rightarrow \infty} \frac{\log n(r, \theta, \varepsilon, a)}{\log r}=\rho
$$

Received March 5, 2006; Revised March 31, 2007.

2000 Mathematics Subject Classification. Primary 30D30, 30D35. zeros.

Key words and phrases. Borel direction, hyper order, the exponent of convergence of

This work was financially supported by NNSF of China 10471048 and NSF of Xianning University KT0623, KZ0629. 
holds for all $a \in \mathbb{C}_{\infty}$ with at most two exceptions, where $n(r, \theta, \varepsilon, a)$ is the number of zeros of $f(z)-a$ in $\{z: \theta-\varepsilon<\arg z<\theta+\varepsilon\} \cap\{0<|z|<r\}$, counting with multiplicities(see [9]). It's well known that every $\rho(\rho>0)$ order meromorphic function has at least one Borel direction(see [12]).

For the study of the differential equation

$$
f^{\prime \prime}+A(z) f=0
$$

where $A(z)$ is an entire function, S. J. Wu [9] investigated the angular distribution of zeros of solutions of $(1)$. In order to state his results, we recall the following definitions. Let $f(z)$ be an entire function in the complex plane and let $\arg z=\theta \in \mathbb{R}$ be a ray. We denote, for each $\varepsilon>0$, the exponent of convergence of zero-sequence of $f(z)$ in the angular region $\{z: \theta-\varepsilon<\arg z<\theta+\varepsilon,|z|>0\}$ by $\lambda_{\theta, \varepsilon}(f)=\limsup _{r \rightarrow \infty} \frac{\log n(r, \theta, \varepsilon, f=0)}{\log r}$ and the second order exponent of convergence of zero-sequence of $f(z)$ by $\lambda_{2, \theta, \varepsilon}(f)=\limsup _{r \rightarrow \infty} \frac{\log \log n(r, \theta, \varepsilon, f=0)}{\log r}$. Furthermore, denote $\lambda_{\theta}(f)=\lim _{\varepsilon \rightarrow 0} \lambda_{\theta, \varepsilon}(f)$ and $\lambda_{2, \theta}(f) \stackrel{r \rightarrow \infty}{=} \lim _{\varepsilon \rightarrow 0} \lambda_{2, \theta, \varepsilon}(f)$.

In [9], S. J. Wu proved the following results.

Theorem 1.1. Let $A(z)$ be a polynomial of degree $n \geq 1$ and let $f_{1}, f_{2}$ be two linearly independent solutions of (1). Set $E=f_{1} f_{2}$ and $\rho=\frac{n+2}{2}$, then $L: \arg z=\theta$ is a Borel direction of order $\rho$ of $E$, if and only if $\lambda_{\theta}(E)=\rho$.

Theorem 1.2. Let $A(z)$ be a transcendental entire function of finite order in the plane and let $f_{1}, f_{2}$ be two linearly independent solutions of (1). Set $E=$ $f_{1} f_{2}$. Suppose that the exponent of convergence of zero-sequence $\lambda(E)=\infty$, then $L: \arg z=\theta_{0}$ is a Borel direction of infinity order of $E$, if and only if $\lambda_{\theta_{0}}(E)=\infty$.

For $n \geq 2$, we consider a linȩar differential equation

$$
f^{(n)}+A_{n-2}(z) f^{(n-2)}+\cdots+A_{1}(z) f^{\prime}+A_{0}(z) f=0,
$$

where $A_{0}(z), \ldots, A_{n-2}(z)$ are entire functions. A classical result, due to Wittich, tells that all solutions of (2) are of finite order of growth if and only if all coefficients $A_{0}(z), \ldots, A_{n-2}(z)$ are polynomials. In this paper, we study the equation (2) under the condition some (or all) of the coefficients are transcendental and all of coefficients are finite order growth.

Here a question arises: Let $f_{1}, f_{2}, \ldots, f_{n}$ be $n$ linearly independent solutions of (2). Set $E=f_{1} f_{2} \cdots f_{n}$. Under the condition of $\lambda(E)=\infty$, does we can obtain that $\lambda_{\theta}(E)=\infty$ is equivalent to $L: \arg z=\theta$ is a Borel direction of infinity order of $E$ ?

In this paper, we prove some results concerning the above question. Now there exists a new question: how to describe precisely the properties of growth of solutions of infinite order of (2)? It is to make use of hyper order (see [5]). Let us recall the following definition. 
Definition (see [5]). The order of an entire function $f$ is defined by

$$
\sigma(f)=\limsup _{r \rightarrow \infty} \frac{\log T(r, f)}{\log r}=\limsup _{r \rightarrow \infty} \frac{\log \log M(r, f)}{\log r},
$$

and the hyper order of an entire function $f$ is defined by

$$
\sigma_{2}(f)=\limsup _{r \rightarrow \infty} \frac{\log \log T(r, f)}{\log r}=\limsup _{r \rightarrow \infty} \frac{\log \log \log M(r, f)}{\log r} .
$$

Where $T(r, f)$ is the Nevanlinna's characteristic function of $f$ and $M(r, f)=$ $\max _{|z|=r}|f(z)|$.

Definition. A ray $L: \arg z=\theta$ is called a Borel direction of hyper order $\rho(0<\rho<\infty)$ of $f$ which has the hyper order $\rho$, if no matter how small the positive number $0<\varepsilon<\pi / 2$ is, for each value $a \in \mathbb{C}_{\infty}$, holds

$$
\limsup _{r \rightarrow \infty} \frac{\log \log n(r, \theta, \varepsilon, a)}{\log r}=\rho,
$$

with at most two exceptional values $a$.

We are now in the position to state our main results.

Theorem 1.3. Let $f_{1}, f_{2}, \ldots, f_{n}$ be $n$ linearly independent solutions of (2). Set $E=f_{1} f_{2} \cdots f_{n}$. Suppose that $\lambda(E)=\infty$ and $E$ is an entire function of hyper order $\rho$, then $\lambda_{2, \theta}(E)=\rho$ if and only if $L: \arg z=\theta$ is a Borel direction of hyper order $\rho$ of $E$.

For the second order differential equation (1), we have

Corollary 1.4. Under the condition of Theorem 1.2, Suppose that $E$ is an entire function of hyper order $\rho$, then $\lambda_{2, \theta}(E)=\rho$ if and only if $L: \arg z=\theta$ is a Borel direction of hyper order $\rho$ of $E$.

It's obvious that Corollary 1.4 is a precise version of theorem 1.2 in the case of $\sigma_{2}(E)=p>0$. As an application of Theorem 1.3, we pose the following theorem. Firstly, we recall the definition of the upper densities of a set $F \subset$ $[0,+\infty)$. We define the linear measure of $F$ by $m(F)=\int_{0}^{+\infty} \chi_{F}(t) d t$, where $\chi_{F}(t)$ is the characteristic function of $F$. The upper densities of $F$ is defined by

$$
\overline{d e n s} F=\limsup _{r \rightarrow+\infty} \frac{m(F \cap[0, r])}{r} .
$$

Theorem 1.5. Let $H$ be a set of complex numbers satisfies $\overline{\text { dens }}\{|z|: z \in H\}>$ 0 , and let $A_{0}(z), \ldots, A_{n-2}(z)$ be the entire function with $\max _{1 \leq k \leq n-2} \sigma\left(A_{k}\right) \leq$ $\sigma\left(A_{0}\right)=\sigma<\frac{1}{2}$, such that for some real constants $0 \leq \beta<\alpha$, we have $\left|A_{0}(z)\right| \geq \exp \left(\alpha|z|^{\sigma-\varepsilon}\right)$ and $\left|A_{k}(z)\right| \leq \exp \left(\beta|z|^{\sigma-\varepsilon}\right), k=1,2, \ldots, n-2$, as $z \rightarrow \infty$ for $z \in H$. Again Let $f_{1}, f_{2}, \ldots, f_{n}$ be $n$ linearly independent solutions of (2). Set $E=f_{1} f_{2} \cdots f_{n}$. Suppose that $\sigma_{2}(E)=\sigma$, then $\lambda_{2, \theta}(E)=\sigma$ if and only if $L: \arg z=\theta$ is a Borel direction of hyper order $\sigma$ of $E$. 
Remark 1.6. Under the condition of Theorem 1.5, Benharrat [1] has proved that every solution $f \neq 0$ of equation (2) satisfies $\sigma(f)=+\infty$ and $\sigma_{2}(f)=\sigma$. On the other hand, under condition of Theorem 1.5, J. Langley [7] proved that every solution $f \neq 0$ of equation (2) satisfies $\lambda(f)=+\infty$. From these claims and Theorem 1.3, Theorem 1.5 follows.

\section{Proof of Theorem}

Our proof requires the Nevanlimna theory in an angular domain. For sake of convenience, we recall some notations and definitions in Nevanlinna's work [8]. Let $f(z)$ be a meromorphic function. Consider a direction $L: \arg z=\theta_{0}$ and an angular domain $\alpha=\theta_{0}-\eta \leq \arg z \leq \theta_{0}+\eta=\beta, \quad 0<\eta<\frac{\pi}{2}$ and for $r>1$ define

$$
\begin{gathered}
A_{\alpha \beta}(r, f)=\frac{k}{\pi} \int_{1}^{r}\left(\frac{1}{t^{k}}-\frac{t^{k}}{r^{2 k}}\right)\left\{\log ^{+}\left|f\left(t e^{i \alpha}\right)\right|+\log ^{+}\left|f\left(t e^{i \beta}\right)\right|\right\} \frac{d t}{t} \\
B_{\alpha \beta}(r, f)=\frac{2 k}{\pi r^{k}} \int_{\alpha}^{\beta} \log ^{+}\left|f\left(t e^{i \theta}\right)\right| \sin k(\theta-\alpha) d \theta \\
C(r, f)=C_{\alpha \beta}(r, f)=2 \sum_{b_{v} \in \triangle}\left(\frac{1}{\left|b_{v}\right|^{k}}-\frac{\left|b_{v}\right|^{k}}{r^{2 k}}\right) \sin k\left(\beta_{v}-\alpha\right),
\end{gathered}
$$

where $k=\frac{\pi}{\beta-\alpha}$ and the summation $\sum_{b_{v} \in \Delta}$ is taken over all the poles $b_{v}=\left|b_{v}\right| e^{i \theta}$ of the function $f(z)$ in the sector $\triangle: 1 \leq|z|<r, \quad \alpha<\arg z<\beta$. Each pole $b_{v}$ occurs in the sum $\sum_{b_{v} \in \triangle}$ as many times as its multiplicity. Otherwise, when pole $b$ occurs in the sum $\sum_{b_{v} \in \Delta}$ only once, we denote it by $\bar{C}(r, f)$. Furthermore, for $r>1$, we define

$$
D_{\alpha \beta}(r, f)=A_{\alpha \beta}(r, f)+B_{\alpha \beta}(r, f), \quad S_{\alpha \beta}(r, f)=C_{\alpha \beta}(r, f)+D_{\alpha \beta}(r, f) .
$$

For sake of simplicity, we omit the subscript in all notations and use $A(r, f)$, $B(r, f), C(r, f), D(r, f)$ and $S(r, f)$ instead of $A_{\alpha, \beta}(r, f), B_{\alpha, \beta}(r, f), C_{\alpha, \beta}(r, f)$, $D_{\alpha, \beta}(r, f)$ and $S_{\alpha, \beta}(r, f)$.

If the meromorphic function $f(z)$ is nonconstant, then for any value $a$, we have

$$
S\left(r, \frac{1}{f-a}\right)=S(r, f)+O(1)
$$

for any $r>1$. This is the first fundamental theorem for $S(r, f)$ (see [10]).

In order to state the second fundamental theorem, it's convenient to introduce the following notations. Denote $C(r, \infty)=C(r, f)$ and for a finite value $a \in \mathbb{C}, C(r, a)=C\left(r, \frac{1}{f-a}\right)$. Then, the second fundamental theorem for $S(r, f)$ may be stated as follows (see [2]): 
Let $f(z)$ be a nonconstant meromorphic function and $a_{j}(j=1,2, \ldots, q$, $q \geq 3$ ) be $q$ distinct values, finite or infinite, then, for $r>1$, we have the inequality

$$
(q-2) S(r, f)<\sum_{j=1}^{q} \bar{C}\left(r, a_{j}\right)+h(r)
$$

where

$$
h(r)=D\left(r, \frac{f^{\prime}}{f}\right)+\sum_{1 \leq j \leq q, a_{j} \neq \infty} D\left(r, \frac{f^{\prime}}{f-a_{j}}\right)+O(1) .
$$

Using the lemma 1, in L. Yang and C. C. Yang [13], we have

$$
D\left(r, \frac{f^{\prime}}{f}\right)= \begin{cases}O(1), \quad \text { when the order of } f(z) \text { is finite, } \\ O\{\log r S(r, f)\}, & \text { otherwise, }\end{cases}
$$

In the latter case, the inequality holds for all the positive value $r$ except a set with finite measure. Hence the second fundamental theorem for $S(r, f)$ can be reduced by

$$
(q-2) S(r, f)<\sum_{j=1}^{q} \bar{C}\left(r, a_{j}\right)+O\{\log r S(r, f)\},
$$

except a set of value $r$ with finite measure.

In order to prove our main results, we need the following Lemma.

Lemma 2.1 (see [10]). With the above notations, let $g(z)$ be a nonconstant meromorphic function and $\Omega(\alpha, \beta)$ be a sector, where $0<\beta-\alpha \leq 2 \pi$, then, for any $r<R$,

$$
\begin{gathered}
A_{\alpha \beta}\left(r, \frac{g^{\prime}}{g}\right) \leq K\left\{\left(\frac{R}{r}\right)^{k} \int_{1}^{R} \frac{\log T(t, g)}{t^{1+k}} d t+\log \frac{r}{R-r}+\log \frac{R}{r}+1\right\}, \\
B_{\alpha \beta}\left(r, \frac{g^{\prime}}{g}\right) \leq \frac{4 k}{r^{k}} m\left(r, \frac{g^{\prime}}{g}\right),
\end{gathered}
$$

where $K$ is a positive constant not depending on $r$ and $R$.

We are now in the position to prove Theorem 1.3.

Proof. We shall prove Theorem 1.3 through the following four steps.

Step 1: Firstly, under the above notations, we have, for any sufficiently small $\varepsilon>0$, on the angular $\theta-\varepsilon<\arg z<\theta+\varepsilon$,

$$
S(r, E)=C\left(r, \frac{1}{E}\right)+O(1) .
$$

It follows from [11] that $\sigma_{2}(E) \leq \sigma$. Here, we give only an out-line of the proof of it. Suppose that $f(z)$ is a non-trivial solution of equation (2), then

$$
\frac{f^{(n)}}{f}+A_{n-2} \frac{f^{(n-2)}}{f}+\cdots+A_{1} \frac{f^{\prime}}{f}+A_{0}=0 .
$$


By Wiman-Valiron theory, there exist a set $D_{1} \subset[1,+\infty)$ which has finite logarithmic measure. We have, when $|z|=r \notin D_{1}$ and $|f(z)|=M(r, f)$,

$$
\frac{f^{k}(z)}{f(z)}=\left(\frac{\nu(r)}{z}\right)^{k}(1+O(1)), k=1,2, \ldots, n,
$$

where $\nu(r)$ denotes the central index of $f$. Combining (5) and (6) we have

$$
v(r)^{n}(1+O(1))+v(r)^{n-2} z^{2} A_{n-2}(1+O(1))+\cdots+z^{n} A_{0}=0 .
$$

Let $\sigma=\max _{0 \leq k \leq n-2}\left\{\sigma\left(A_{k}\right)\right\}$, then for any given $\varepsilon^{\prime}>0$, there exist a finite linear measure set $D_{2} \subset[1,+\infty)$, for sufficiently large $r,|z|=r \notin[0,1] \cup D_{2}$, we have,

$$
\left|A_{k}\right| \leq \exp \left\{r^{\sigma+\varepsilon^{\prime}}\right\}, k=1,2, \ldots, n-2 .
$$

The above expression and (7) implies that, for $|z|=r \notin[0,1] \cup D_{1} \cup D_{2}$ and for sufficiently large $r$,

$$
v(r) \leq n r^{n} \exp \left\{r^{\sigma+\varepsilon^{\prime}}\right\} \leq \exp \left\{r^{\sigma+2 \varepsilon^{\prime}}\right\}
$$

Hence

$$
\sigma_{2}(f) \leq \sigma .
$$

It follows from (8) that $\sigma_{2}(E) \leq \sigma$.

Now, we continuously prove the equation (4) by using the similar argument as [11]. Denote the Wronskian of $f_{1}, f_{2}, \ldots, f_{n}$ by $W=W\left(f_{1}, f_{2}, \ldots, f_{n}\right)$. By Proposition 1.4 .8 in [6], we have $W\left(f_{1}, f_{2}, \ldots, f_{n}\right)=C$, where $C$ is a positive constant. On the other hand, we have

$$
\frac{1}{E}=\frac{W}{E} \frac{1}{C}=\frac{1}{C}\left|\begin{array}{cccc}
1 & 1 & \ldots & 1 \\
\frac{f_{1}^{\prime}}{f_{1}} & \frac{f_{2}^{\prime}}{f_{2}} & \ldots & \frac{f_{n}^{\prime}}{f_{n}} \\
\frac{f_{1}^{(n-1)}}{f_{1}} & \frac{f_{2}^{(n-1)}}{f_{2}} & \ldots & \frac{f_{n}^{(n-1)}}{f_{n}}
\end{array}\right| .
$$

Hence, we have

$$
\frac{1}{E}=\frac{1}{C} \sum_{1 \leq i_{l} \neq i_{l} \leq n}(-1)^{\tau} \prod_{l=1}^{n-1} \frac{f_{i_{l}}^{(l)}}{f_{i_{l}}} .
$$

By lemma 2.1, in which, we set $R=2 r$, for sufficiently small $\varepsilon$ and any $f_{i}$ we have

$$
A_{\theta-\varepsilon, \theta+\varepsilon}\left(r, \frac{f_{i}^{\prime}}{f_{i}}\right)=O\left(\int_{1}^{2 r} \frac{\log ^{+} T\left(t, f_{i}\right)}{t^{1+\frac{\pi}{2 \varepsilon}}} d t\right)=O\left(\int_{1}^{2 r} \frac{t^{\sigma+1}}{t^{1+\frac{\pi}{2 \varepsilon}}} d t\right)=O(1) .
$$

Since $m\left(r, \frac{f_{i}^{\prime}}{f_{i}}\right)=O(\log (r T(r, f)))=O\left(r^{\sigma+1}\right)$. We deduce from Lemma 2.1 that

$$
B_{\theta-\varepsilon, \theta+\varepsilon}\left(r, \frac{f_{i}^{\prime}}{f_{i}}\right) \leq \frac{4 k}{r^{k}} m\left(r, \frac{f_{i}^{\prime}}{f_{i}}\right)=O\left(r^{\sigma+1-\frac{\pi}{2 \varepsilon}}\right)=O(1) .
$$


The above two expressions imply $D_{\theta-\varepsilon, \theta+\varepsilon}\left(r, \frac{f_{i}^{\prime}}{f_{i}}\right)=O(1)$. Similarly, we have

$$
\begin{gathered}
D_{\theta-\varepsilon, \theta+\varepsilon}\left(r, \frac{f_{i}^{(h)}}{f_{i}}\right) \leq \sum_{i=1}^{h} D_{\theta-\varepsilon, \theta+\varepsilon}\left(r, \frac{f_{i}^{(l)}}{f_{i}^{(l-1)}}\right)+O(1)=O(1), \\
i=1,2, \ldots, n ; \quad h=2,3, \ldots, n-1 .
\end{gathered}
$$

Therefore, combining (9), we have

$$
D_{\theta-\varepsilon, \theta+\varepsilon}\left(r, \frac{1}{E}\right)=O(1) \text {. }
$$

By the definition and the first fundamental theorem for $S(r, f)$, we can derive (4).

Step 2: We shall prove that, for any $0<\eta<\frac{\pi}{2}$,

$$
\limsup _{r \rightarrow \infty} \frac{\log \log S(r, E)}{\log r} \leq \rho .
$$

In fact, by (3), we have

$$
S(r, E)<\sum_{j=1}^{3} \bar{C}\left(r, a_{j}\right)+O\{\log r S(r, f)\},
$$

holds for any three distinct finite values $a_{j}, j=1,2,3$. Since

$$
\bar{C}\left(r, a_{j}\right) \leq 2 n\left(r, \theta, \eta, a_{j}\right),
$$

and when $R>r>0$, we have

$$
n(r, \theta, \eta, E=a) \leq n(r, E=a) \leq N(R, E=a) \log \frac{r}{R} \leq T(R, E) \log \frac{r}{R} .
$$

Hence for any $\varepsilon>0$, we have $n\left(r, \theta, \eta, a_{j}\right)<\exp \left(r^{\rho+\varepsilon}\right)$. From this we can deduce $\bar{C}\left(r, a_{j}\right)<\exp \left(r^{\rho+\varepsilon}\right)$. Substituting this result into (11), we get (10).

Step 3: In this step, we shall prove the following statement. In order that $L: \arg z=\theta$ is a Borel direction of hyper order $\rho$ of $E$, if and only if for each $\eta\left(0<\eta<\frac{\pi}{2}\right)$, we have

$$
\limsup _{r \rightarrow \infty} \frac{\log \log S(r, E)}{\log r}=\rho .
$$

Assume that $L$ is a Borel direction of the function $E$ of hyper order $\rho$, if for some $\eta\left(0<\eta<\frac{\pi}{2}\right)$, we have

$$
\limsup _{r \rightarrow \infty} \frac{\log \log S(r, E)}{\log r}<\rho .
$$

By the first fundamental theorem of $S(r, E)$, for any finite value $a$, we have $S\left(r, \frac{1}{E-a}\right)=S(r, E)+O(1)$. Since $C(r, a) \leq S\left(r, \frac{1}{E-a}\right)$, then

$$
C(r, a) \leq S\left(r, \frac{1}{E-a}\right)=S(r, E)+O(1) .
$$


On the other hand,

$$
\begin{aligned}
C(2 r, a) & \geq C_{\theta-\frac{\eta}{2}, \theta+\frac{\eta}{2}}(2 r, a) \\
& \geq 2 \sum_{1<\left|b_{v}\right|<r, \theta-\frac{\eta}{2}<\beta_{v}<\theta+\frac{\eta}{2}}\left(\frac{1}{\left|b_{v}\right|^{k}}-\frac{\left|b_{v}\right|^{k}}{(2 r)^{2 k}}\right) \sin k\left(\beta_{v}-\theta+\frac{\eta}{2}\right) \\
& \geq 2 \sum_{1<\left|b_{v}\right|<r, \theta-\frac{\eta}{3}<\beta_{v}<\theta+\frac{\eta}{3}}\left(\frac{1}{\left|b_{v}\right|^{k}}-\frac{\left|b_{v}\right|^{k}}{(2 r)^{2 k}}\right) \sin k\left(\beta_{v}-\theta+\frac{\eta}{2}\right),
\end{aligned}
$$

where $k=\frac{\pi}{\eta}$. In the sector $\triangle: 1<|b|<r, \theta-\frac{\eta}{3}<\beta<\theta+\frac{\eta}{3}$, we have $0<\frac{\eta}{6}<\beta_{v}-\theta+\frac{\eta}{2}<\frac{5 \eta}{6}<\frac{\pi}{2}$. We write a sum of above expression as a Stieltjes-integral and the partial integration of the above Stieltjes-integrals now results in

$$
\begin{aligned}
C(2 r, a) \geq & \int_{1}^{r} \frac{1}{t^{k}} d n(t)+\frac{1}{(2 r)^{2 k}} \int_{1}^{r} t^{k} d n(t) \\
\geq & k \int_{1}^{r} \frac{1}{t^{k+1}} n(t) d t+\frac{n(r)}{r^{k}}-\frac{r^{k} n(r)}{r^{2 k}} \\
& +\frac{k^{2 k}}{(2 r)^{2 k}} \int_{1}^{r} t^{k-1} n(t) d t \\
\geq & \frac{n(r)}{r^{k}}-\frac{r^{k} n(r)}{(2 r)^{2 k}} \\
\geq & \left(1-\frac{1}{2^{2 k}}\right) \frac{n(r)}{r^{k}}
\end{aligned}
$$

where a short-hand notation $n(t)=n\left(t, \theta, \frac{\eta}{3}, a\right)$ will be used. Substituting (14) to (13) and combining (12), we get

$$
\limsup _{r \rightarrow \infty} \frac{\log \log n\left(r, \theta, \frac{\eta}{3}, a\right)}{\log r}<\rho .
$$

Since $a$ is arbitrary, the above expression is incompatible with the hypothesis that $L$ is a Borel direction of hyper order $\rho$ of $E$.

Conversely, assume that for any $\eta\left(0<\eta<\frac{\pi}{2}\right)$, we have

$$
\limsup _{r \rightarrow \infty} \frac{\log \log S(r, E)}{\log r}=\rho \text {. }
$$

Suppose that $L$ is not a Borel direction of hyper order $\rho$ of $E$. Then there exist a $\eta$ and three distinct values $a_{j} \in \mathbb{C}_{\infty}(j=1,2,3)$, such that for sufficiently large $r$, we have

$$
n\left(r, \theta, \eta, a_{j}\right)<\exp \left(r^{\rho^{\prime}}\right)\left(\rho^{\prime}<\rho\right)
$$

For the three distinct value $a_{j}$, we have

$$
C\left(r, a_{j}\right) \leq 2 n\left(r, \theta, \eta, a_{j}\right) .
$$

We deduce from (16), (17) and (3) that, when $r$ is sufficiently large, we have $S(r, E)<\exp \left(r^{\rho^{\prime}}\right)$. Hence, we get a contradiction.

Step 4: We prove that $\lambda_{2, \theta_{0}}(E)=\rho$, if and only if for each $\eta\left(0<\eta<\frac{\pi}{2}\right)$, we have

$$
\limsup _{r \rightarrow \infty} \frac{\log \log S(r, E)}{\log r}=\rho .
$$


Assume that $\lambda_{2, \theta_{0}}(E)=\rho$. If there is a $\eta\left(0<\eta<\frac{\pi}{2}\right)$, for which, we have

$$
\limsup _{r \rightarrow \infty} \frac{\log \log S(r, E)}{\log r}<\rho .
$$

As we did in the proof of (15), we have

$$
\limsup _{r \rightarrow \infty} \frac{\log \log n\left(r, \theta, \frac{\eta}{3}, a\right)}{\log r}<\rho .
$$

Hence

$$
\lambda_{2, \theta_{0}, \frac{\eta}{3}}(E)<\rho .
$$

For any $0<\varepsilon<\frac{\eta}{3}$, we can derive $\lambda_{2, \theta_{0}, \varepsilon}(E)<\rho$. As $\varepsilon$ can be arbitrary small, we obtain $\lambda_{2, \theta_{0}}(E)<\rho$. This result is incompatible with the hypothesis.

Conversely, assume that for any $\eta\left(0<\eta<\frac{\pi}{2}\right)$, we have

$$
\limsup _{r \rightarrow \infty} \frac{\log \log S(r, f)}{\log r}=\rho
$$

Combining (4) and (18), we can deduce that, for any $\eta$,

$$
\limsup _{r \rightarrow \infty} \frac{\log \log n\left(r, \theta_{0}, \eta, E=0\right)}{\log r} \geq \rho .
$$

This expression implies that $\lambda_{2, \theta_{0}}(E) \geq \rho$. On the other hand, $E$ is an entire function of hyper order $\rho$, we have $\lambda_{2, \theta_{0}}(E) \leq \rho$. The proof of step 4 is now complete.

Combining the step 3 and the step 4 , Theorem 1.3 follows.

From the proof of Theorem 1.3, we have the following corollaries.

Corollary 2.2. Suppose that $f(z)$ is an entire function of hyper order $\rho$, with above notations, in order that $L: \arg z=\theta_{0}$ is a Borel direction of hyper order $\rho$ of the function $f(z)$, if and only if for each $\eta\left(0<\eta<\frac{\pi}{2}\right)$, we have

$$
\limsup _{r \rightarrow \infty} \frac{\log \log S(r, f)}{\log r}=\rho .
$$

Corollary 2.3. Suppose that $f(z)$ is an entire function of hyper order $\rho$, with above notations, if $\lambda_{2, \theta}(f)=\sigma$, it is necessary that for each $\eta\left(0<\eta<\frac{\pi}{2}\right)$, we have

$$
\limsup _{r \rightarrow \infty} \frac{\log \log S(r, f)}{\log r}=\rho
$$

Furthermore, the ray $L: \arg z=\theta$ is a Borel direction of hyper order $\rho$ of $f(z)$. 


\section{References}

[1] B. Belaïdi, Estimation of the hyper-order of entire solutions of complex linear ordinary differential equations whose coefficients are entire functions, Electron. J. Qual. Theory Differ. Equ. 2002 (2002), no. 5, 1-8.

[2] C. T. Chuang, On Borel directions of meromorphic functions of infinite order. II, Bull. Hong Kong Math. Soc. 2 (1999), no. 2, 305-323.

[3] H. Guo, J. H. Zheng, and T. W. Ng, On a new singular direction of meromorphic functions, Bull. Austral. Math. Soc. 69 (2004), no. 2, 277-287.

[4] W. K. Hayman, Meromorphic functions, Oxford Mathematical Monographs Clarendon Press, Oxford 1964.

[5] K. H. Kwon, On the growth of entire functions satisfying second order linear differential equations, Bull. Korean Math. Soc. 33 (1996), no. 3, 487-496.

[6] I. Laine, Nevanlinna theory and complex differential equations, de Gruyter Studies in Mathematics, 15. Walter de Gruyter \& Co., Berlin, 1993.

[7] J. K. Langley, Some oscillation theorems for higher order linear differential equations with entire coefficients of small growth, Results Math. 20 (1991), no. 1-2, 517-529.

[8] R. Nevanlinna, Über die Eigenschaften meromorpher Funktionen in einem Winkelraum, Acta Soc. Sci. Fenn. 50 (1925), no. 12, 1-45.

[9] S. J. Wu, Angular distribution in complex oscillation theory, Sci. China Ser. A 48 (2005), no. $1,107-114$.

$[10] \_$, On the location of zeros of solutions of $f^{\prime \prime}+A(z) f=0$ where $A(z)$ is entire, Math. Scand. 74 (1994), no. 2, 293-312.

[11] C. F. Yi, Angular distribution of solutions of a higher-order differential equation, Acta Math. Sinica (Chin. Ser.) 48 (2005), no. 1, 133-140.

[12] L. Yang, Value distribution theory, Translated and revised from the 1982 Chinese original. Springer-Verlag, Berlin; Science Press, Beijing, 1993.

[13] L. Yang and C. C. Yang, Angular distribution of values of $f f^{\prime}$, Sci. China Ser. A 37 (1994), no. 3, 284-294.

[14] J. H. Zheng, On transcendental meromorphic functions with radially distributed values, Sci. China Ser. A 47 (2004), no. 3, 401-416.

ZHAOJUN WU

DEPARTMENT OF MATHEMATICS

XiANNING UNIVERSITY

Xianning, 437100, P. R. China

CURRENT ADDRESS:

SCHOOL OF MATHEMATICS

South China Normal University

Guangzhou 510631, P. R. China

E-mail address: wuzj52@hotmail.com

DAOCHUN SUN

SCHOOL OF MATHEMATics

SOUTh ChINA Normal UNIVERSITY

Guangzhou 510631, P. R. China

E-mail address: sundch@scnu.edu.cn 